\title{
Wireless Broadband Access with the Application of IEEE 802.11b based Wi-Fi Model
}

\author{
Aditi Daund \\ Digital communication \\ research scholars Sagar Institute of \\ science\&Technology \\ gandhinagar ,Bhopal(m.p)
}

\author{
Pankaj Kumar Vyas \\ Associate professor \\ Sagar Institute of \\ science\&Technology \\ gandhinagar ,Bhopal(m.p)
}

\begin{abstract}
Wireless Broadband Access service is now a day's more popular for data transmission. Traditional high-speed broadband solutions rely upon wired technologies specifically digital subscriber line (DSL). Wi-Fi is helpful in providing any type of connectivity such as the fixed or portable connectivity while not the need of LoS (Line of Sight) of the base station. Mobile Broadband Wireless Network (MBWN) could be a versatile and economical answer for remote areas where wired technology and additionally terminal quality can't be provided. The IEEE 802.11 family based Wi-Fi is the most promising technologies for broadband wireless metropolitan area networks (WMANs) and these are capable of providing high output even on long distances with varied QoS. In this paper the basic physical layer architecture is discussed and on the basis of the study a new Wi-Fi model is developed for supporting the wireless broadband access.
\end{abstract}

\section{Keywords}

Digital Subscriber Line(DSL), Line of Site (LoS), Broadband wireless access (BWA), Point-to Multipoint (P2MP), PLCP,PPDU etc..

\section{INTRODUCTION}

Mobile and Wireless Communication and high speed data communications have enormously enhanced the way business is conducted since the new millennium. The technology permits workers, partners, and customers to access company data from almost anywhere and anytime. The necessity for universal data access, combined with inflated employee productivity and effectiveness is driving the demand for enterprise mobile applications. The growth of wireless broadband networks is anticipated to step by step outmatch landline communications as advancements in these technologies are facultative higher broadband speeds. The expansion in broadband wireless networks may be attributed to high demand for wireless transmission services admire voice, data, video and also the development of recent wireless standards. There are lots of alternative driving factors, that are self-addressed elsewhere that have led to the speedy and continuous amendment of the wireless networks worldwide. Quality could be a major driver for mobile broadband networks as a result of mobile professionals still demand access to their company networks remotely anyplace and anytime. The ever growing want for mobile web access, interactive services, training, and entertainment; the necessity for one commonplace for seamless roaming; ability across networks; and upward integration of earlier wireless network technologies are driving factors for brand new developments in wireless networks.

Generally, a broadband wireless network is categorized into two types: fixed and mobile wireless as shown in figure 1 .
The broadband fixed wireless network technologies of interest here are wireless fidelity (Wi-Fi), that is an IEEE 802.11 standard and worldwide interoperability for Microwave Access (WiMax) that is additionally an IEEE 802.16 standard. The two broadband mobile wireless network technologies are the third Generation (3G) and Fourth Generation (4G) networks. The 3G standards are outlined by ITU-T, IMT2000 and also the standards for the $4 \mathrm{G}$ are currently being defined.

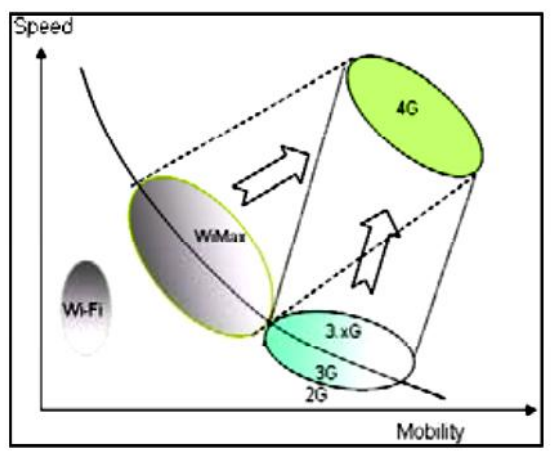

Figure 1: Fixed and Wireless Mobile Network

\section{BACKGROUND OF BWA}

Over the past few decades, mobile and wireless communications as well as web are the foremost profound and necessary technology in data technologies that quickly grow and endlessly amendment human life. It looks that these "twins" unbroken developing in an exceedingly parallel and freelance ways in which. Specifically, the information theory, i.e. Shannon Theory, is taken into account together of the foremost elementary theory in communications [1], whereas web evolves in apply from laboratory to globalization $[2,3]$. Nonetheless, recent years, the demands of mobile data keep proliferating, meanwhile, the smart phone and mobile applications become more and more powerful and multifarious. These result in a rare chance to mix each mobile communications and web, i.e. mobile web. Mobile web allows U.S. to get pleasure from numerous web services through mobile and wireless accessing reminiscent of on-line video, file sharing, net browsing, voice service, and etc. Consequently, mobile and wireless network (MWN) is dynamic from voice-centric to multimedia-oriented $[4,5]$. 
Unfortunately, traditional mobile and wireless network will hardly maintain with this trend and presently face many refractory challenges:

- The contradiction between the increasing scarce spectrum and also the poor resource utilization [6] aggravates step by step.

- There are tremendous heterogeneous wireless networks, however it's tough to realize economical interworking between them to fulfill end users' diversified expectations whereas making certain the existence of numerous heterogeneous networks [7].

- Tightly coupling with specific hardware and lack of versatile control interfaces, this mobile network will hardly offer a quick track for technological innovations.

- Mobile services and applications prominently become multifarious, and that they need differing types of network characteristics [8]. Unfortunately, traditional MWN solely support these multifarious services with identical network characteristic. As a result, it naturally deteriorates the quality of service $(\mathrm{QoS})$ and quality of experience $(\mathrm{QoE})$ of end users.

- Carriers sink into a difficulty that the network prices continue to grow whereas the revenues stay stagnate, that is inconsistent with the service and application proliferation $[9,10]$.

These challenges directly have an effect on all the participants as well as domain, carriers and finish users. Consequently, they impede the evolution of future MWN.

Most of those pressing challenges are deeply rooted within the inherent design of the present mobile communication and web. for example, vertical-constructing methodology adopted by mobile communication hampers the convergence of heterogeneous networks; the distributed and independent operating model leads to low resource utilization; the tightly coupling between hardware and wireless protocols makes MWNs tough to evolve and deploy new technologies and functions; mobility becomes more and more difficult and hard to handle as a result of IP address based web is oriented towards the fixed network from its birth. Consequently, merely evolving the present mobile networks will hardly meet such great expectations while not fundamental architectural changes. On the opposite hand, although clean-slate design is vital for facultative the networking field to mature into a real discipline [11], it's entirely incompatible with the present technologies and should not be acceptable due to the incalculable prices. Therefore, a revolutionary design is needed to alter the MWN to possess the potential to handle these pressing challenges and to realize smoothly evolution.

Software-defined network (SDN) [12, 13], an innovative paradigm, is one in all the most recent and hottest topics in networks. SDN advocates decoupling the control plane and data plane of networks, that dramatically simplifies network control and allows innovation and evolution by abstracting the control functions of the network into a logically centralized control plane. Network devices become straightforward packet forwarding and process devices (the data plane) which will be programmed via an open interface [14], such as Open Flow $[15,16]$. Significantly, in recent years, academia and industry lead one new trend that extending SDN to mobile and wireless networks, i.e. software-defined wireless network (SDWN). Familiarizing towards the expectations and challenges of MWN, SDWN benefits many aspects of MWN, for example wireless resource optimizing, convergence of heterogeneous networks, fine-grained controllability, and efficient programmability for network innovation and smooth evolution. However, key technologies in software defined wireless networks (SDWN) are still need to be self-addressed, such as the end-to-end architecture, physical layer software system process, control ways, and etc.

Network virtualization $[17,18]$ is presently attracting present attention from both academia and industry. It allows multiple concurrent virtual networks to run on shared substrate resources. Network virtualization separates the traditional internet service providers (ISPs) into two new roles: infrastructure network providers (InPs) and service providers (SPs) [19] and, consequently, SPs need virtual networks to supply varied services by leasing resources from InPs. Recent years, some researchers target wireless network virtualization (WNV). It considerably improves the resource utilization, edges the network innovations, offers the made-to-order services, and enhances the QoS and QoE.

With the rapid growth of mobile demands and also the ever increasing diversity of services and applications, mobile internet has been an irresistible trend. On the opposite hand, software-defined network and network virtualization emerge and provide a good chance to integrate communications and web. Though software-defined wireless network and wireless network virtualization are thought of as totally different technologies, they extremely complement one another. It's quite necessary to mix SDWN and WNV to expeditiously address the challenges and supply opportunities for future mobile and wireless network, as an instance the Network function Virtualization (NFV) cluster [20, 21].

\section{IEEE 802.11 FAMILY PHYSICAL LAYER ARCHITECTURE}

This section focuses on operation of items specified by the 802.11 series of standards for the physical layer. These items will include the PLCP and PMD sublayers, management layer entities, and generic management primitives. An in-depth understanding of how the physical layer operates and how it interfaces with the MAC layer is vitally important to the analyst's understanding of information gathered by a wireless protocol analyzer.

\subsection{PLCP Sub layer}

The MAC layer communicates with the Physical Layer Convergence Protocol (PLCP) sublayer via primitives (a set of "instructive commands" or "fundamental instructions") through a service access point (SAP). When the MAC layer instructs it to do so, the PLCP prepares MAC protocol data units (MPDUs) for transmission. The PLCP minimizes the dependence of the MAC layer on the PMD sublayer by mapping MPDUs into a frame format suitable for transmission by the PMD. The PLCP also delivers incoming frames from the wireless medium to the MAC layer. The PLCP sublayer is illustrated in Figure 1. 


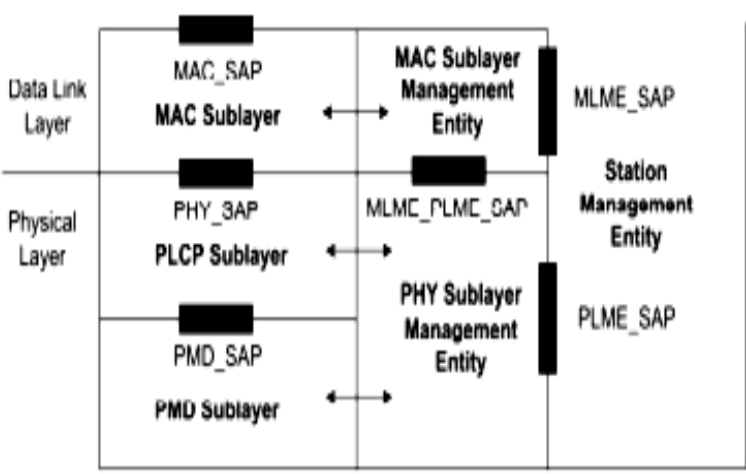

Figure 1: 802.11 Physical and MAC Layer Architecture

The PLCP appends a PHY-specific preamble and header fields to the MPDU that contain information needed by the Physical layer transmitters and receivers. The 802.11 standard refers to this composite frame (the MPDU with an additional PLCP preamble and header) as a PLCP protocol data unit (PPDU). The MPDU is also called the PLCP Service Data Unit (PSDU), and is typically referred to as such when referencing physical layer operations. The frame structure of a PPDU provides for asynchronous transfer of PSDUs between stations. As a result, the receiving station's Physical layer must synchronize its circuitry to each individual incoming frame.

\subsection{PMD Sublayer}

Under the direction of the PLCP, the Physical Medium Dependent (PMD) sublayer provides transmission and reception of Physical layer data units between two stations via the wireless medium. To provide this service, the PMD interfaces directly with the wireless medium (that is, RF in the air) and provides modulation and demodulation of the frame transmissions. The PLCP and PMD sublayers communicate via primitives, through a SAP, to govern the transmission and reception functions.

\subsection{Management Layer Entities}

Both MAC and PHY layers conceptually include management entities, called the MAC sublayer management entity and the PHY sublayer management entity. These entities are referred to as the MAC Layer Management Entity (MLME), and the Physical Layer Management Entity (PLME). These entities provide the layer management service interfaces through which layer management functions may be invoked. In order to provide correct MAC operation, a station management entity (SME) shall be present within each station. The SME is a layer-independent entity that may be viewed as residing in a separate management plane or as residing "off to the side." The exact functions of the SME are not specified in the 802.11 standard, but in general this entity may be viewed as being responsible for such functions as the gathering of layer dependent status from the various layer management entities, and similarly setting the value of layer-specific parameters. The SME would typically perform such functions on behalf of general system management entities and would implement standard management protocols.

\subsection{Generic Management Primitives}

The management information specific to each layer is represented as a management information base (MIB) for that layer. The MAC and PHY layer management entities are viewed as "containing" the MIB for that layer. The generic model of MIB-related management primitives exchanged across the management SAPs is to allow the SAP user-entity to either GET the value of a MIB attribute, or to SET the value of a MIB attribute. The practical usage example of management primitives is when the user configures an access point or a mobile station's wireless utilities. This is done through a configuration interface such as CLI, GUI, SNMP, or custom software. Configuration of the access point's features through its web interface, for example, will SET a MIB attribute value to perhaps true/false or to some logical value.

\section{IEEE 802.11B BASED BWA SYSTEM}

A Wi-Fi mesh-networking infrastructure is a wireless network within which a set of 802.11a (b or g-based) nodes are interconnected and share the communication protocols. This topology extends the range of LANs and WLANs. WMN repeatedly maintain dynamic path and configurations by learning on its own by making a path for exchanging information with one another. In WMN, nodes are put in throughout the neighborhood space (within a school/college campus) where the small nodes serve as simple routers. Each node transmits a signal to neighboring nodes, which in turn transmits signal to consecutive node so on. This method is continual till the data reaches its destination. In WMN, blocked subscribers will indirectly access the base station through alternative nodes. Nodes will amendment location during this design and may even be superimposed or removed. These networks are applicable wherever the devices got to be a lot of mobile and wireless such as in future business so that self-configuration is easy. The advantages of WMN include balanced traffic, robustness, resilience, low initial prices, accessibility, quality and spatial reuse. Comparatively WMN can dynamically adapt to interference not like the single-hop networks that are loaded down with nodes.

Benefits of using Wi-Fi for fixed-access solutions:

- Availability of off-the-shelf 802.11 products

- Small deployments want less investment initially

- More flexible over wired installations

The IEEE 802.11b Direct Spread Spectrum (DSSS) physical layer is used for the BWA in the Wi-Fi. As discussed in previous section the trans-receiver system is developed for 1 , 2, 5.5, and $11 \mathrm{Mbps}$ rates in $2.4 \mathrm{GHz}$ ISM band. Figure 2 shows the construction of DSSS PLCP protocol Data Unit, which includes a long preamble, the header and MPDU (PSDU) as specified in 802.11 standards. For synchronization of incoming signal with receiver preamble play main role. Preamble synchronizes properly before the actual content of the frame arrives. The header is used for providing information about the frame, and the PSDU is the MPDU the transmitting station is sending. Figure 3 shows the Simulink model based on figure 2. The $802.11 \mathrm{~b}$ standard further specifies rates 5.5 and $11 \mathrm{Mbps}$, each using Complementary Code Keying (CCK) modulation. For giving two configuration option administrations have to provide a short preamble. 


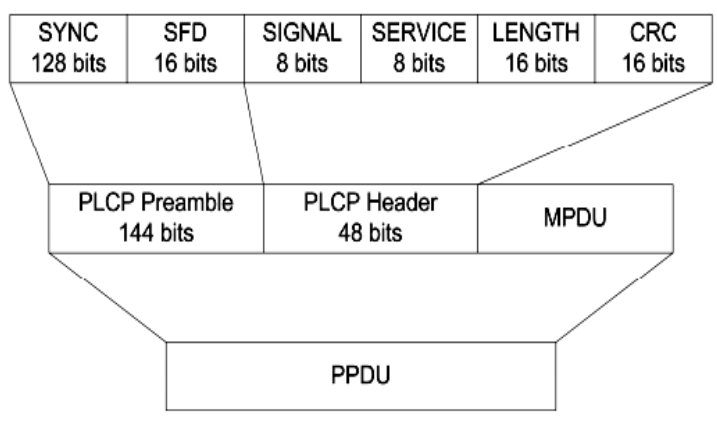

Figure 2: DSSS based PLCP Protocol Data Unit

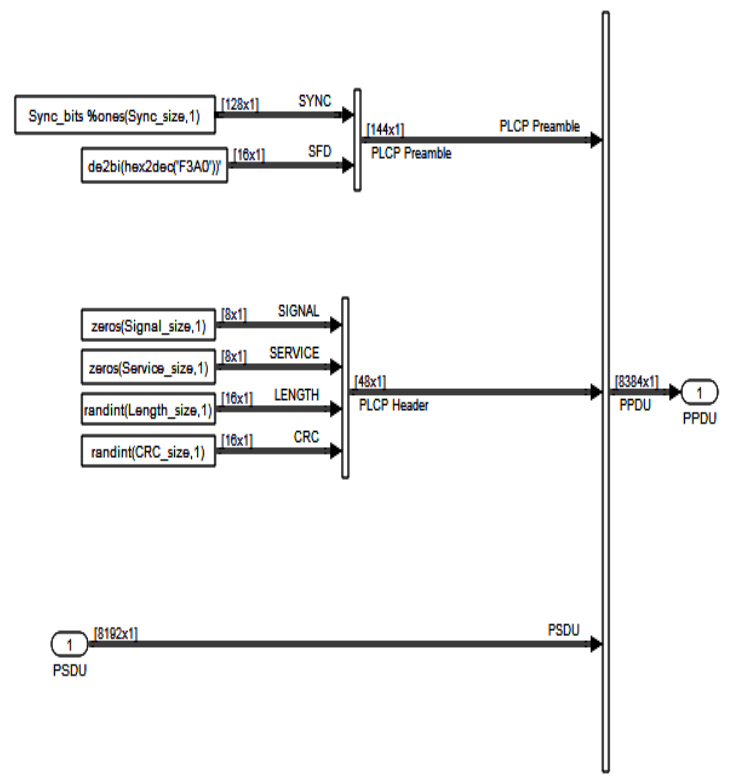

Figure 3: Simulink model of PLCP Protocol Data Unit

\section{RESULTS}

The benefits of IEEE 802.11 family are used for making in trans-receiver system for Wi-Fi system to enhance the data sending and receiving system. The whole work is simulated in MATALB software for verification of the work. The model is run for $1,2,5.5,11 \mathrm{Mbps}$ data in wireless broadband access. Figure 4 shows the BER vs. ES/NO for all speed.

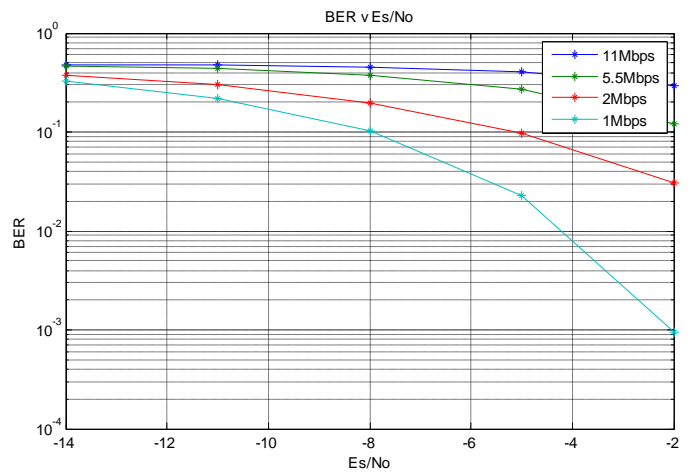

Figure 4: Performance Analysis of Broadband Wireless Access based Wi-Fi model for different Speed

The simulation is based on the DBSK and DQPSK based system. Figure 5 shows the $1 \mathrm{Mbps}$ based received system.
Rest of figure shows the 2, 5.5, 11Mbps based trans-receiver system received signal with or without short preamble.

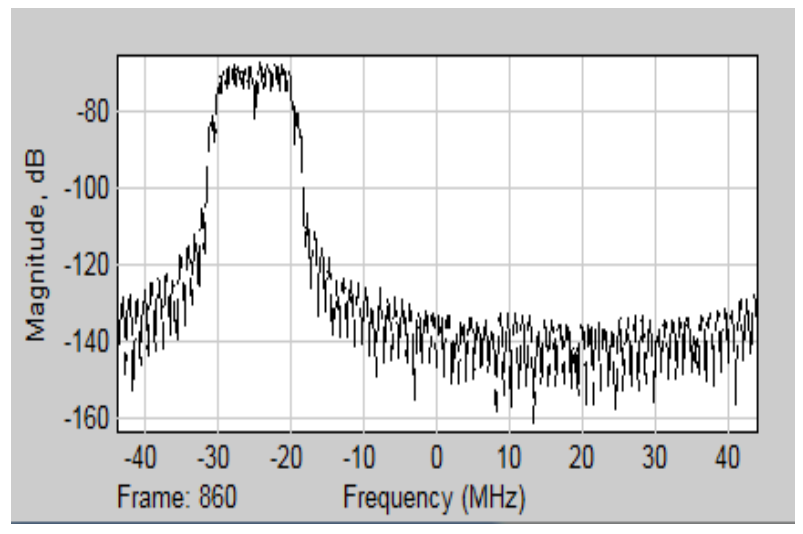

Figure 5: Spectrum plot of Received Signal at 1Mbps Speed with DBPSK Modulation

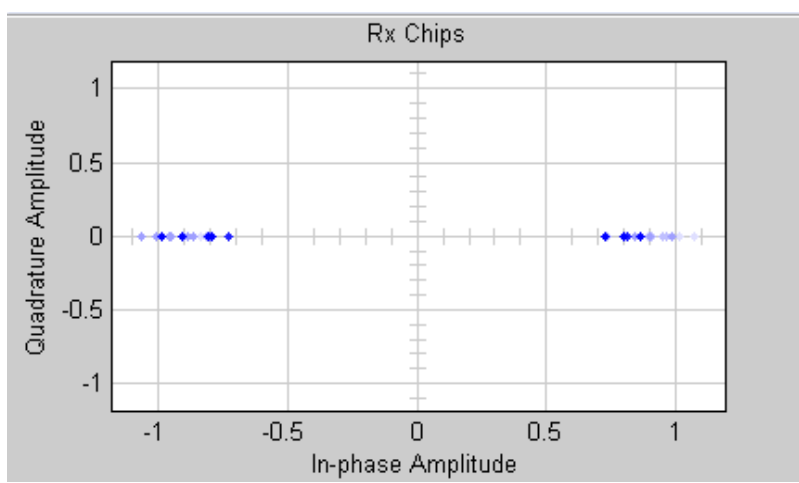

Figure 6: Scatter plot of the Received Signal at 1 Mbps Speed by DBPSK modulation

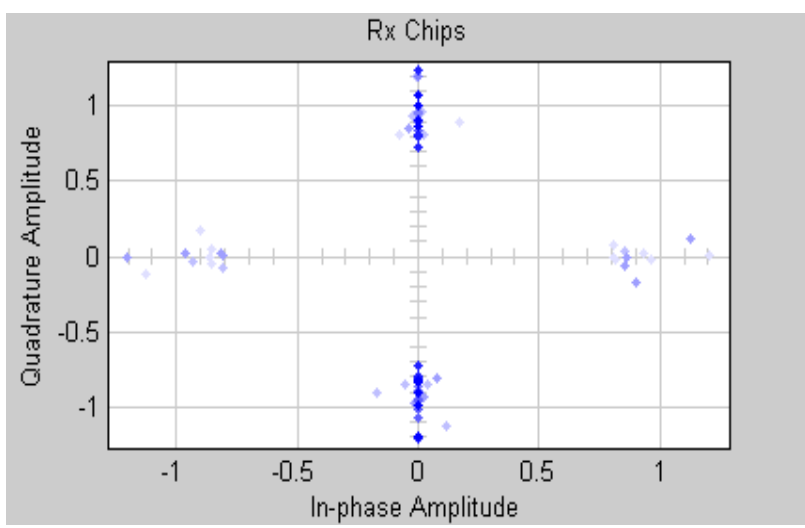

Figure 7: Scatter plot of the Received Signal at 2 Mbps Speed by DQPSK modulation without short preamble

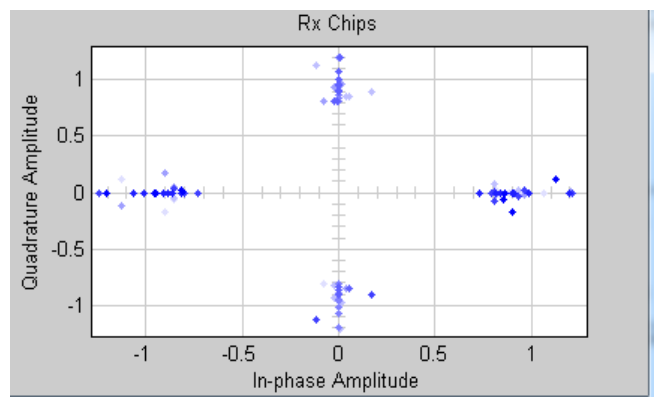

Figure 8: Scatter plot of the Received Signal at 2 Mbps Speed by DQPSK modulation without short preamble 


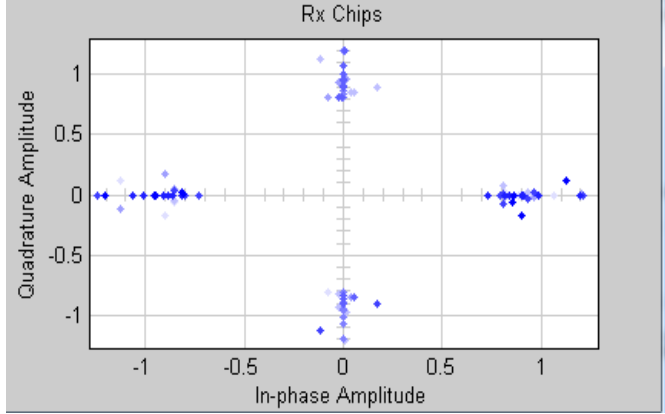

Figure 9: Scatter plot of the Received Signal at 2 Mbps Speed by DQPSK modulation with short preamble

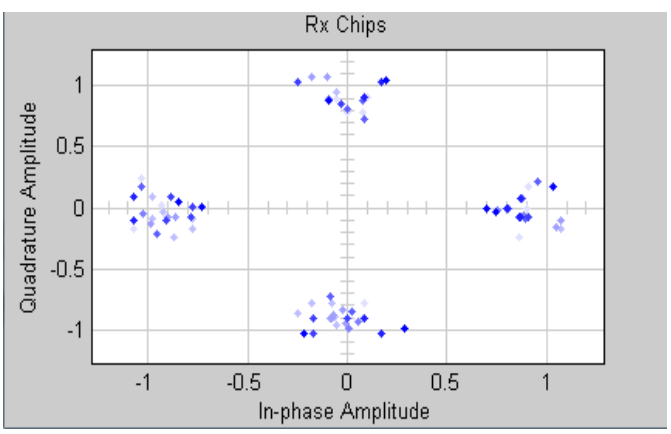

Figure 10: Scatter plot of the Received Signal at $5.5 \mathrm{Mbps}$ Speed by DQPSK modulation without short preamble

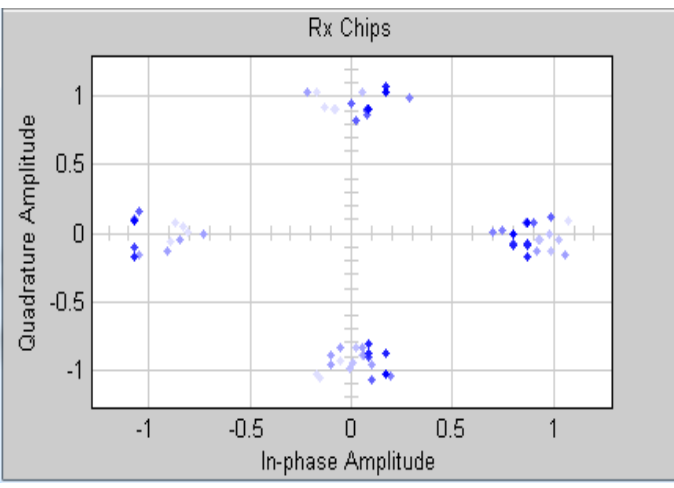

Figure 11: Scatter plot of the Received Signal at 5.5 Mbps Speed by DQPSK modulation with short preamble

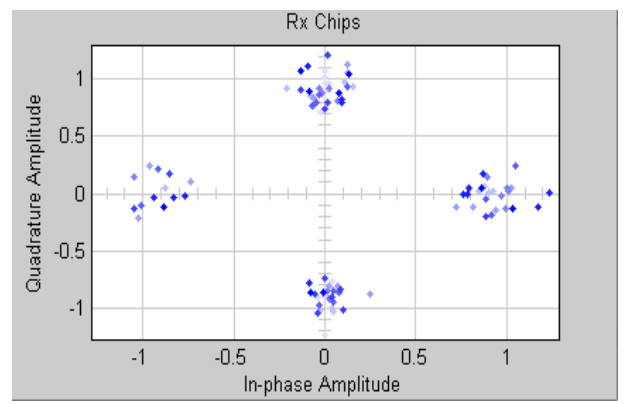

Figure 12: Scatter plot of the Received Signal at 11 Mbps Speed by DQPSK modulation without short preamble

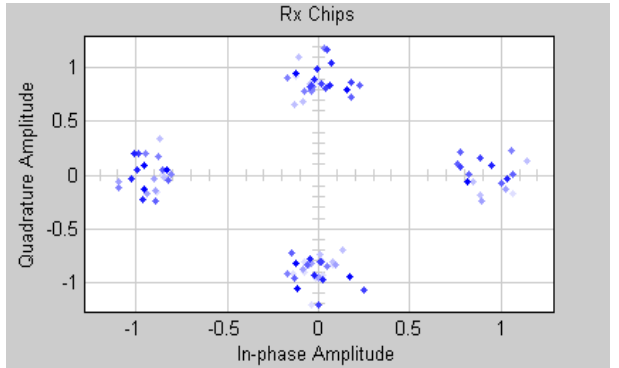

Figure 13: Scatter plot of the Received Signal at 11 Mbps Speed by DQPSK modulation with short preamble

\section{CONCLUSION}

Broadband wireless access is emerging technology used for enhancement of the data speed in mobile communication. The key contribution of this paper is implementation of PHY layer of IEEE 802.11 family based Wi-Fi system. In this paper discussed the background of the wireless communication. On the basis of background speeding the data is necessary for mobile communication. The $\mathrm{Wi}-\mathrm{Fi}$ is the solution for increasing the speed of the data. IEEE802.11 is used for making of the model. The model is rum in MATLAB software. Results shows the verification of the proposed work.

\section{REFERENCES}

[1]. Cover TM, Thomas JA (2006) Elements of information theory. 2nd Edn, (Wiley series in telecommunications and signal processing). Wiley, New Jersey

[2]. Kleinrock L (2010) An early history of the internet [history of communications]. IEEE Commun Mag 48(8):26-36

[3]. Leiner BM, Cerf VG, Clark DD, Kahn RE, Kleinrock L, Lynch DC, Postel J, Roberts LG, Wolff S (2009) A brief history of the internet. SIGCOMM Comput Commun Rev 39(5):22-31

[4]. Karjaluoto H (2007) An investigation of third generation $(3 \mathrm{~g})$ mobile technologies and services. Contemp Manag Res 2(2):91-104

[5]. Kennedy M, Ksentini A, Hadjadj-Aoul Y, Muntean G (2013) Adaptive energy optimization in multimediacentric wireless devices: a survey. IEEE Commun Surv Tutor 15(2):768-786

[6]. Yucek T, Arslan H (2009) A survey of spectrum sensing algorithms for cognitive radio applications. IEEE Commun Surv Tutor 11(1):116-130

[7]. Ferrus R, Sallent O, Agusti R (2010) Interworking in heterogeneous wireless networks: comprehensive framework and future trends. IEEE Wirel Commun 17(2):22-31

[8]. Stankiewicz R, Jajszczyk A (2011) A survey of qoe assurance in converged networks. Comput Netw 55(7):1459-1473

[9]. Marsch P, Raaf B, Szufarska A, Mogensen P, Guan H, Farber M, Redana S, Pedersen K, Kolding T (2012) Future mobile communication networks: challenges in the design and operation. IEEE Veh Technol Mag $7(1): 16-23$

[10].Fehske A, Fettweis G, Malmodin J, Biczok G (2011) The global footprint of mobile communications: the 
ecological and economic perspective. IEEE Commun Mag 49(8):55-62

[11].Rexford J, Dovrolis C (2010) Future internet architecture: cleanslate versus evolutionary research. ACM Commun 53(9):36-40

[12]. Yu M, Rexford J, Freedman MJ, Wang J (2010) Scalable flowbased networking with difane. SIGCOMM Comput Commun Rev 41(4):351-362

[13].Kim H, Feamster N (2013) Improving network management with software defined networking. IEEE Commun Mag 51(2):114-119

[14]. Astuto BN, Mendon M, Nguyen XN, Obraczka K, Turletti T (2014) A survey of software-defined networking: past, present, and future of programmable networks. IEEE Commun Surv Tutor 16(3):1617-1634

[15].Lara A, Kolasani A, Ramamurthy B (2013) Network innovation using open flow: a survey. IEEE Commun Surv Tutor 99:1-20

[16]. McKeown N, Anderson T, Balakrishnan H, Parulkar G, Peterson L, Rexford J, Shenker S, Turner J (2008) Openflow: enabling innovation in campus networks. SIGCOMM Comput Commun Rev 38(2):69-74

[17]. Chowdhury NMK, Boutaba R (2010) A survey of network virtualization. Comput Netw 54(5):862-876

[18]. Feamster N, Gao L, Rexford J (2007) How to lease the internet in your spare time. SIGCOMM Comput Commun Rev 37(1):61-64

[19]. Chowdhury N, Boutaba R (2009) Network virtualization: state of the art and research challenges. IEEE Commun Mag 47(7):20-26
[20].ETSI (2012) Network functions virtualisation white paper. http://portal.etsi.org/NFV/NFV White Paper.pdf. Accessed Oct 2012

[21].Jain R (2013) Network virtualization and software defined networking for cloud computing: a survey. IEEE Commun Mag 51(11):24-31

[22].Ericsson (2012) Mobile report on the pulse of the networked society. Ericsson Mobility Report. http://www.ericsson.com/res/docs/2012/ericssonmobility-report-november-2012.pdf. Accessed Nov 2012

[23].Banerjee A, Chen X, Erman J, Gopalakrishnan V, Lee S, Merwe J (2013) Moca: A lightweight mobile cloud offloading architecture.

[24].In: Proceedings of the 8th ACM international workshop on mobility in the evolving internet architecture, ser. MobiArch '13, New York, pp 11-16

[25]. Chen BX (2012) Carriers warn of crisis in mobile spectrum. The New York Times. http://www.nytimes.com/2012/04/18/technology/mobilecarriers-warn-of-spectrum-crisis-others-see-hyper bole.html?pagewanted=all. Accessed April 2012

[26]. Research R (2010) Mobile broadband capacity constraints and the need for optimization. http://www.rysavy.com/Articles/2010 02 Rysavy Mobile Broadband Capacity Constraints.pdf. Accessed Feb 201

[27].Open network foundation (onf) (2013). https://www.opennet

[28].working.org/. 